\title{
Validity and Reliability of the Instrument for Assessing the Child's Constitution (ICC): Individualising Processes in Diagnosing Children With a Developmental Disorder
}

\author{
M.H. Niemeijer ${ }^{1,2}$, E.W. Baars ${ }^{1,3}$, J. Hoekman ${ }^{1,4}$ \& A.J.J.M. Ruijssenaars ${ }^{5}$ \\ ${ }^{1}$ University of Applied Sciences, Leiden, the Netherlands \\ ${ }^{2}$ Kingfisher Foundation, Driebergen/Zeist, the Netherlands \\ ${ }^{3}$ Louis Bolk Institute, Bunnik, the Netherlands \\ ${ }^{4}$ University Leiden, Leiden, the Netherlands \\ ${ }^{5}$ Department of Special Needs Education and Youth Care, University of Groningen, the Netherlands
}

Correspondence: Niemeijer, M.H., University of Applied Sciences, Leiden; Kingfisher Foundation, Driebergen/Zeist, the Netherlands.

Received: November 21, 2018

Accepted: December 14, 2018

Online Published: December 28, 2018

doi:10.20849/ijsn.v3i3.531

URL: https://doi.org/10.20849/ijsn.v3i3.531

\begin{abstract}
In children developmental disorders expresses themselves by a variable presentation of symptoms and frequent manifestation of comorbidity of a complex nature. In addition to the classification of a disorder, individualising diagnostics is a means to realize assessment and treatment that are fine-tuned to the child.

In the day to day care of children with a developmental disorder the Instrument for assessing the Child's Constitution (ICC) is a supporting means to map out the child's individual situation. The instrument is based on an anthroposophic anthropology and typology. It provides an individualised diagnosis by mapping the degree of (dis)balance in child development. In three developmental fields, the cognitive, the affective and the conative field, the (dis)balance of functioning is assessed by the ICC. The instrument consists of two parts. With reference to the three fields part I consists of 36 items, each having a subscale with 12 polar formulated items on a seven-point scale. For each of the three fields part II includes a visual analogue scale (VAS). The results of Part I and II are made comparable by putting them both on a ten-point scale. The results are graphically represented as a profile of the constitution.

In this article we present the results of a study with the ICC on psychometric properties in a research group $(n=535)$ and a control group $(n=148)$. As reference of the assessment of the quality of the ICC a Consensus-based Standard for the selection of health Measurement Instruments is used.
\end{abstract}

Keywords: developmental disorders, individual assessment, child constitution, anthroposophic anthropology, typology

\section{Introduction}

\subsection{Developmental Disorders}

Developmental disorders include a wide range of disorders in relation to mental and physical functions in which children differ from what is considered to be a normal development. These 'neurodevelopmental disorders' (DSM-5, American Psychiatric Association, 2013) pervasively interfere in the development, are accompanied with problems and disorders in bio-psychosocial functions which permanently influence the child's biography (Rutter, 2011). In the DSM-5 the following items are considered to be developmental disorders: Intellectual Disabilities, Communication Disorders and Specific learning, Motor Disorders, Autism Spectrum Disorder (ASD) and Attention-Deficit/Hyperactivity Disorder (ADHD). A traumatic attachment disorder that has been acquired at an early age may be considered as one of the 'Other specified Neurodevelopmental Disorders' (APA, 2015; Vander Kolk, 2017; Coock et al., 2017). The Multiple complex Developmental Disorder (McDD) is often considered as a sub-type of ASD (Oranje, Lahuis, Van Engeland, Van der Gaag, \& Kemner, 2013). As a 
developmental disorder (Buitelaar \& Van Gaag, 1988), characterised by social impairments, affective dysregulation and thought disturbance, McDD is not separately mentioned in the DSM-5.

The aetiology of the developmental disorders is multifunctional. Hereditary diseases, genetic and chromosome deviations are well-known causes. Furthermore there are risk factors that may disturb the development from conception until childhood years and may result in disorders that are hard to be clearly classified (Pennington, 2006). In these a-typically neurobiological dysfunctions and disorders comorbidity and heterogeneity regularly occur for which the terms of 'co-occurrence' and 'continuum' are used (Kaplan, Crawford, Cantell, Kooistra \& Dewey, 2006).

Because of the complexity in which developmental disorders in children occur, diagnostics are usually individualised. (Rutter, 2011). To do this, and in addition to the classification of descriptive diagnostics, the coherence and the seriousness of the child's problems are stated with hypotheses about causes and origin. Dimensional diagnostics are a means to attribute a quantative dimension to the actual seriousness of the developmental disorder. (Hudziak, Achenback, Althoff \& Pine, 2007; Kraemer, 2007). In the DSM-5 (2013) a dimensionalisation has been introduced for the first time by specifying the 'current severity' of the neurodevelopmental disorders in 'mild', 'moderate' and 'severe' (APA, 2013). Various instruments based on a dimensional approach are used for screening and diagnostics. This holds good for example for the CBCL (Achenbach, 1991; Avision, 2014), for the ADHD questionnaire (Sherman, Iacono \& McGue, 1997) and for the SDQ (Goodman \& Goodman, 2009).

\subsection{Constitution}

In anthroposophical inspired care of children with developmental disorders the constitution is assessed as part of a diagnosis that is focused on the individual situation of the child. This approach has been derived from an existing typology of the concept of constitution. (Steiner, 1985) In later years this approach has been further developed and drawn up as a method according to social and scientific understandings. (Holzapfel, 1985; Niemeijer; Baars, 2005; Grimm \& Kaschubowski, 2008; Niemeijer \& Gastkemper, 2009; Göschel, 2012)

The concept of constitution is derived from the Latin 'constituere' meaning 'to build'. In typology it is used in an active form, as a verb. In medical literature the concept refers to someone's disposition, the genetic constitution. This is held responsible for the development of the general make-up and functioning of the organism. Over the past few years the plasticity of living organisms under the influence of epigenetic reactions on circumstantial influences has increasingly been recognized (Hochberg et al., 2010; Gluckman, Hanson \& Low, 2011). This results in a more dynamic character of the concept of constitution. In constitutional typology the term 'constitution' is used in the meaning of the basic pattern of bio-psychosocial functioning. This pattern is not static. It changes during one's life. It is determined by one's inherited general make-up, a relatively static factor. As dynamic factors there are the epigenetic effects caused by circumstantial influences and one's own personality. This combination of static and dynamic factors means that the constitution may be influenced and with this it is also open to therapy or treatment.

\subsection{Typology}

In the typology of the constitution three fields of development can be distinguished, the fields of cognitive, affective conative functioning (Hilgard, 1980; Pervin \& John, 1999). Each of these three fields is in support of specific bio-psychosocial functions of respectively observation and thinking, feeling and experiencing, acting and behaviour. Research into the functioning in this 'trilogy of mind' (Hilgard, 1980) is part of diagnostics of children with developmental problems (Dai \& Sternberg, 2004; Hengeveld \& Schudel, 2004; Dennis et al., 2013).

In each of the three developmental fields an ordering principle is active which constitutes the bio-psychosocial functioning. These principles are: 'Modelling' in the cognitive field, 'Connecting' in the affective field and 'Moving' in the conative field. For all bio-psychosocial functioning these ordering principles create a prototypical situation of balance of 'good' and 'normal'. For example the thyroid gland: the prototypical balance of the thyroid gland is the eu-thyreoid functioning. Imbalance in the function is expressed by a spectrum, in a certain one-sidedness, as either hyper- or hypo-thyreoidism. Such a spectrum in which an ordering principle can be expressed is also known in psychology, for instance in the continuum between the poles introvert-extravert and internalising-externalising. Constitutional typology fits in with the latter spectrums. This typology is based on centripetal versus centrifugal dynamics in ordering principles as holds good for internalising versus externalising 
Centripetal versus centrifugal dynamics in the Modelling principle results in a spectrum between the polar one-sidedness that is characterised as 'Densifying/Obsessive' and 'Dissolving/Forgetful'; as to the Connecting principle in the polar one-sidedness 'Closed-up/Clenched' and 'Open/Flowing-out'; as to Moving principle characterised as 'Decelerating/Heavy' and 'Accelerating/Light' (Niemeijer, Baars, Hoekman \& Ruijssenaars, 2018).

Exemplary instances of imbalance in which an ordering principle can express itself for the modelling principle is coherence in thinking, thought formation, with a possible imbalance of 'hooking up in', versus 'digressing from one's own subject of conversation'. Imbalance in connective dynamics may lead to 'closed' versus 'open in contact'. An imbalance in moving can express itself as 'lacks initiative', versus 'many and variegated initiatives'. A practitioner can assess to what extent the effects of the three principles expresses itself as observable phenomena. Assessment of the constitution is given as either having a balance or one-sidedness, imbalance, on a continuum between one-sidedness situations on both sides of an optimal prototypical central position.

With this approach constitutional typology coincides with the idea in which health is more and more considered as a dynamic process, defined as 'the power to adept and to rule oneself in the light of the physical, emotional and social challenges in life' (Huber et al. 2011). One's individual health depends on an interplay of healthprotecting and health-threatening factors and is influenced by powers of adaption and one's own resilience (Luthar, Sawyer \& Brown. 2006)

\subsection{The Instrument}

The ICC consists of two parts. In two ways the extent of the (im)balance in the child's functioning in the three developmental domains can be assessed. Part I consists of 36 items, each field having a subscale with 12 polar formulated items on a seven-point scale. Part I is meant for completion by health professionals. For each of the three fields Part II includes a VAS-scale that is completed by the practitioner who is in charge for the diagnosis. In both, the item scales and the VAS scales, it is about a qualitative assessment which is represented on a seven-point scale and a VAS scale respectively. Studies about the development of the ICC have been published before (Niemeijer \& Baars, 2004; Niemeijer, Baars, Hoekman \& Ruijssenaars, 2018).

The target group in which the ICC can be used in clinical practice, consists of children and youths that are examined because of developmental problems. The ICC is available to practitioners on a website with a user's name and a pass word. On this website they can find further information. The ICC on the website is completed by the practitioner who is in charge of the child, a doctor or a psychologist. As additional data the following is asked: the date of birth of the child, the diagnostic classification that has been determined, the cognitive level of functioning and the setting in which the child has been taken care of. The time that is needed for completion is about 15 minutes.

The results of Part I and II are made comparable by putting them both on a ten-point scale. These are graphically represented as a profile of the constitution. The results give a profile of the child's constitution which consists of the scores of Part I and II. This profile shows the extent of the (im)balance in cognitive, affective and conative functioning. This constitutional profile provides the practitioner with tools for interventions in the fields of health and development promotion and of symptom and complaint control (Niemeijer \& Gastkemper, 2009).

\section{Research}

The research is focused on determining the psychometric properties of the ICC: the intra- and inter-rater reliability, internal consistency, content validity, criterion validity and structure validity of the ICC. As reference of the assessment of the quality of the ICC the Consensus-based Standards for the selection of health Measurement Instruments is used (COSMIN) (Mokkink et al., 2012).

\subsection{Research Group}

Research has been done in two groups of children and youngsters, the research group and the control group. The research group consists of children/youngsters whose ICC is entered because of developmental problems or a developmental disorder for which they are getting special care in health care institutions in the Netherlands. Data have been received from these children by means of ICC's which were completed by the practitioner. See: www.louisbolk.nl/constitutieinstrument

The yougsters-from the age of twelve-and their parents were informed by the practitioner of the health care institution about the results of the diagnostic research, about the constitutional profile as defined by the ICC and about the possible classification of a developmental disorder. Based on these results agreements were made about coaching and treatment. 
Including criteria of the research were age, minimum age 4 years, maximum age 23 years and a maximum of 3 missing values in the completed ICC. The children whose instruments have been completed on the website and who meet these criteria are included in the research.

The control group consists of children from two school classes and of children from employees of an institution for mentally handicapped and child and youth psychiatry. These parents responded to a call for participation which was sent to them via email. The parents completed Part I of the ICC.

\subsection{Research Methods}

\subsubsection{Reliability}

The number of missing values is counted for the separate items and the VAS scales. At a percentage of $<5 \%$ the item is accepted (Schafer, 1999) for the ICC and with all analyses the missing values are substituted by the average of the item concerned.

The intra-(test - retest) and inter-rater reliability are calculated by determining the Pearson correlations between pairs of observation for each of the three subscales of Part I of the ICC. In order to determine the intra-rater reliability the same person is asked to complete Part I of the ICC at moment 0 and after two weeks. The inter-rater reliability is determined by completion of Part I of the ICC by two people, independent of one another.

Internal consistency is assessed by determining Cronbach's alpha, the extent to which the 12 items of each subscale of Part I of the ICC are related.

\subsubsection{Validity}

Regarding content validity the question is answered if the items of each domain in Part I and if the polar descriptions in the VAS scales in Part II represent adequate and valid realizations of the constitutional typology. Professionals selected by the researchers for their experience assess the extent of importance of the items on a five-point scale and of the VAS scales as operational effects of the ordering principles for the three bio-psychosocial domains.

The construct validity is assessed by determining the convergence between the outcome of Part I and Part II of the ICC. This is calculated as Pearson-correlation standard, the extent of similarity in the completed instruments between the three subscales in Part I and the three VAS scales in Part II of the ICC. In the same way the coherence between the results of Part I of the ICC and the CBCL has been assessed.

Besides the ICC 74 children have been subjected to the CBCL. It is expected that coherence is found between the extent of seriousness and the nature of problematic behaviour, the latter in relation to inter- and externalizing problems respectively. The CBCL comprises problem scales that refer to 'internalising problems'; other problem scales refer to the 'externalising' problems. The seriousness of the problem assessed with CBCL has been compared with the absolute values of the 36 item scores of Part I of the ICC, the extent to which each of the scores deviates from 0 . As to the assessment of the coherence regarding the nature of the problematic behaviour mini-scores were added and compared with internalising behaviour. The plus-scores all taken together were compared with externalising behaviour.

The structural validity is assessed with a Principle Component Analysis (PCA) with Varimax rotation and gives information about the extent to which the three subscales of Part I of the ICC appear as independent unities in the empirical data.

The standard score has been assessed by subjecting children without a classified developmental disorder to Part I of the ICC. To this end two schools were approached and after an information meeting with parents and teachers Part I was completed by the parents of 148 children.

\section{Results}

\subsection{Characteristics of the Research Groups}

The research group consists of 535 children and youngsters in the age bracket of 4 to 23 years. The control group consists of children without a classified developmental disorder. In total 148 children. Data about the children are stated in Table 1. 
Table 1. Number of children/youngsters, gender division and average age

\begin{tabular}{lcccccc}
\hline \multicolumn{2}{c}{ Children with a developmental disorder } & \multicolumn{3}{c}{ In control group } \\
& Number (Perc.) & Aver. & Std.Dev. & Number & Aver. & Std.Dev. \\
\hline Female & $177(33 \%)$ & 11,1 & 4,3 & $89(60 \%)$ & 9.3 & .1 .3 \\
Male & $358(67 \%)$ & 10,5 & 3,8 & $59(40 \%)$ & 9.6 & 1.6 \\
In total & 535 & 10,7 & 4.0 & 148 & 9.4 & 1.4 \\
\hline
\end{tabular}

The diagnostic classification at the moment of completion of the TCC and the percentages of preventing the next disorder has been assessed for ASS, AD(H)D, Reactive Attachment Disorder, Down-syndrome and Multi complex Developmental Disorder (McDD). As other classifications: mood and behavioural disturbances, epilepsy, spasticity and some syndromes with an intellectual disability. Table 2 shows an overview of the classifications for girls and boys.

Table 2. Diagnostic classification and gender division in the research group

\begin{tabular}{llcccc}
\hline & & Number & Female & Male & Percent \\
\hline Class. & ASD & 133 & 24 & 109 & 24,9 \\
& ADHD & 74 & 13 & 61 & 13,8 \\
& RHS & 62 & 26 & 36 & 11,6 \\
& McDD & 29 & 6 & 23 & 5,4 \\
& DS & 33 & 16 & 17 & 6,2 \\
& Other & 52 & 23 & 29 & 9,7 \\
None & 152 & 69 & 83 & 28,4 \\
In total & 535 & 177 & 358 & 100 \\
\hline
\end{tabular}

The section 'Other' comprises all classifications that differ from the five that were mentioned (ASD, ADHD, RHS, McDD, DS)

The children's and youngsters' level of cognitive functioning varied from average, inclusive of above average, to seriously intellectual disabled. Table 3 shows the relation between the cognitive functioning and the developmental disorders.

Table 3. Level of functioning in relation to the developmental disorders

\begin{tabular}{|c|c|c|c|c|c|c|c|}
\hline & ASD & ADHD & RHS & McDD & DS & Others & Total (Perc.) \\
\hline Average to above av. & 20 & 32 & 6 & 12 & 0 & 50 & $120(22.4)$ \\
\hline Weakly gifted & 35 & 21 & 18 & 9 & 0 & 35 & $118(22.1)$ \\
\hline Mild ID & 43 & 20 & 31 & 8 & 0 & 38 & $140(26.2)$ \\
\hline Moderate ID & 19 & 0 & 7 & 0 & 13 & 35 & $74(13.8)$ \\
\hline Severe ID & 16 & 1 & 0 & 0 & 20 & 46 & $83(15.5)$ \\
\hline Total & 133 & 74 & 62 & 29 & 33 & 204 & $535(100)$ \\
\hline
\end{tabular}

The largest group of children/youngsters was staying in a clinical setting during the research $(51.6 \%)$. In policlinic treatment was $30.5 \%$ and in day care $17.9 \%$ of the children. Table 4 shows an overview of the setting of care in which the children received assistance.

Table 4. Setting of care in which children received assistance

\begin{tabular}{|c|c|c|c|c|c|c|c|c|}
\hline & \multicolumn{8}{|c|}{ Classification } \\
\hline & & ASD & ADHD & RHS & $\mathrm{McDD}$ & DS & Anders & Totaal \\
\hline \multirow[t]{3}{*}{ Setting } & Policlinic & $29(17.8)$ & $48(29.4)$ & $12(7.4)$ & $7(4.3)$ & $1(0.6)$ & $66(40.5)$ & $163(100)$ \\
\hline & Clinic & $69(25.0)$ & $17(6.2)$ & 37 (13.4) & $17(6.2)$ & $31(11.2)$ & $105(38.0)$ & $276(100)$ \\
\hline & Daycare & $35(36.5)$ & $9(9.4)$ & $13(13.5)$ & $5(5.2)$ & $1(1.0)$ & $33(34.4)$ & $96(100)$ \\
\hline Total & & $133(24.9)$ & $74(13.8)$ & $62(11.6)$ & $29(5.4)$ & $33(6.2)$ & $204(38.1)$ & $535(100)$ \\
\hline
\end{tabular}




\subsection{Reliability of the TCC}

\subsubsection{Missing Values}

The complete number of missing values in the items in the entire research group ( $\mathrm{n}=683$ with each 36 items) is $158(0.6 \%)$. The maximum number of missing values for one single item is $20(2.9 \%)$. In all analyses the missing values have been substituted by the average of the item concerned. On the basis of the missing values no items have been left out.

\subsubsection{Inter-rater Reliability}

The inter-rater reliability has been determined by the completion of Part I of the ICC by two independent people with 117 children/youngsters (assessment 1 and 2). The Pearson-correlations between the pairs of observation of each of the three subscales of Part I were: .76 for subscale 1, .63 for subscale 2 and .79 for subscale 3, all significant $(\mathrm{p}>0,01)$ For Part I as a whole the correlation was .81

\subsubsection{Intra-rater Reliability}

With 60 children Part I of the ICC was completed at time 0 and after 2-7 weeks (on average 5.4 weeks) by the same person. The Pearson correlations between the first and the second assessment for the subscales of Part I were: .84 for subscale 1, ' 87 for subscale 2 and .82 for subscale 3, all significant (p.0,01). For Part I in total the correlation was 90 .

\subsubsection{Internal Consistency}

Internal consistency was determined by assessing Cronbach's alpha, the extent to which each of the three domains of Part I (subscales 1, 2, 3) are mutually related. This was determined in the research group of children with developmental disorders ( $\mathrm{N}=535)$. Cronbach's alpha was .66 for subscale 1, .64 for subscale 2 and .63 for subscale 3. Cronbach's alpha for Part I in total was .75.

\subsection{Validity of the ICC}

\subsubsection{Content Validity}

The ICC was submitted to 21 experts: eight remedial education experts/psychologists, seven physicians, three therapists and three group leaders. To each of the 36 polar formulated items and to the three VAS scales they have indicated the level of significance of the three polar constitutional types on a five-point scale. Four items, all from subscale 1 , were assessed on the 5 -point scale $<3.50$. The other 32 items of Part I of the ICC were assessed $>3.50$. The three VAS scales with an explanation how to complete the form were assessed as 4.3, 4.1 and 4.3

\subsubsection{Construct Validity}

The convergent validity as coherence between the three subscales of Part I and the three VAS scales of Part II of the ICC $(\mathrm{N}=535)$ was determined by assessing the Pearson-correlations. The correlation between subscale 1 and VAS 1 was .47. Between subscale 2 and VAS 2.49 and between subscale 3 and VAS 3.61.

The expected coherence between the results of the CBCL and the ICC regarding the seriousness and the nature of the problems was determined by measuring the Pearson-correlations. In Table 5 the Pearson-correlations are shown between the CBCL scores (of the internalising, externalising and total problem score) and the ICC scores (absolute values of the scores when added, i.e. the extent to which the score of each item deviates from $0 \mathrm{~N}=74$.

Table 5. Pearson-correlations between the CBCL scores and the ICC scores

\begin{tabular}{lrrrr}
\hline ICC & subschale1 & subschale2 & subschale3 & Totalscore \\
\hline CBCL & & & & \\
Internalising & $.47^{* * *}$ & $.40^{* *}$ & $.39^{* *}$ & $.45^{* *}$ \\
Externalising & $.54^{* *}$ & $.57^{* *}$ & $.61^{* *}$ & $.62^{* *}$ \\
Total problem score & $.64^{* *}$ & $.62^{* *}$ & $.63^{* *}$ & $.67^{* *}$ \\
\hline
\end{tabular}

\subsubsection{Structure Validity}

By means of Principle Component Analysis with Varimax rotation it has been assessed if the three subscales of the ICC Part I appear in the empirical data as independent unities. In an analysis with three factors the declared variance is $29.2 \%$. Subscale 1 strongly fills in on the third factor. The subscales 2 and 3 show overlap, both fill 
in on the first two factors. An analysis with two factors (23.3\% declared variance) confirms this picture. Subscale 1 fills in on the second factor. Subscales 2 and 3 fills in mainly on the first factor.

\section{Standard Score}

With 148 children without a classified development disorder Part I of the ICC has been completed by the parents. The children involved are pupils of two school in medium-sized towns in the north and the centre of the country and children of employees from an institute of VGZ and GGZ. The average score as is shown in the ICC-profile on a $+/$ - 10-pointscale were respectively -3.3 for subscale $1,+4.4$ for subscale 2 and +4.7 for subscale 3 .

\section{Discussion}

In this practice-oriented research the psychometric properties of the ICC have been investigated, the instrument for assessing the child's constitution. The properties were judged according to the Consensus-based Standards for the selection of health Measurement Instruments (COSMIN-checklist, 2012).

The research group consisted of 535 children/youngsters in the age bracket of 4 to 23 years who get care due to a possible developmental disorder and a control group of 148 children without a confirmed developmental disorder. The COSMIN gives a minimum number of 100 as adequate size of the research group.

The inter-rater and intra-rater reliability of the ICC was assessed by determining the Pearson-correlations. As criterion for this the COSMIN-checklist gives Pearson-correlations $>.70$ as a standard for a positive evaluation. The correlations of the inter-rater reliability of the subscales of Part I $(n=117)$ were $.76, .63$ and .79 and for Part I as a whole.81, all of them significant $(\mathrm{p}<.01)$. For the intra-rater reliability $(\mathrm{n}=60)$ the correlations were $.84, .87$ and .82 , for Part I as a whole .90, all of them significant ( $\mathrm{p}<.01)$

The internal consistency of the three subscales of the ICC Part I ( $n=535)$ was slightly lower with respectively Cronbach's alpha .66, .64 and .63 than what the COSMIN-checklist gives as norm for a good internal consistency: .70 tot.90.

The content validity was researched by interrogating 21 hands-on experts about their judgement of the level of significance of the items and of the explanation that went with the VAS scales in order to assess the construct of the three developmental domains. On a 5-point scale four items of subscale I $<3.50$, which was the norm laid down by the researchers, were evaluated.

The ICC was evaluated by determining construct and structure validity. The construct validity as correlation between the three subscales of part I and the three VAS scales of Part II of the ICC (N=535) was calculated by assessing the Pearson-correlations. The Pearson-correlation between subscale 1 and VAS 1 was .47; between subscale 2 and VAS 2.49 and between subscale 3 and VAS 3.61 . The COSMIN-checklist does not give criteria for this, but it does for criterion validity, >.70, as a standard for the correlation with a 'golden standard'. The correlations that were found between the item lists of Part I and VAS scales in the ICC are low.

The expected coherence between ICC and CBCL as to seriousness and nature of problematic behaviour was investigated by determining the Pearson correlations. The Pearson correlation that was found of .67 appeared to be moderate to good regarding the correlation of the total problem score, with .62 moderate regarding correlations in externalised behaviour and with .45 weak regarding the correlation in internalised behaviour.

The declared variance the Principle Component Analysis with Varimax rotation is limited. In the analysis with three factors as well as in the analysis with two factors the variance remains below $30 \%$. A more important outcome is that subscales 2 and 3 which are related to respectively connecting in the affective domain and moving in the conative field, fill in on the same factors in this analysis.

A 'standard score' was acquired by completion by parents of Part I of the ICC with 148 children. This shows an imbalance: respectively $-3.3,+4.4$ and +4.7 on subscale 1,2 and 3 .

\section{Conclusions}

Based on the COSMIN-criteria the size of the research group is largely sufficient for the research of psychometric properties. The inter-rater as well as the intra-rater reliability is alright. The outcome regarding internal consistency is somewhat below the COSMIN-norm. As to validity the content validity has been judged as good. The expected relation of seriousness and nature of the problems between ICC and CBCL has been confirmed to a reasonable degree.

Three results of the research demand special attention. The results of the ICC indicate that the three subscales of the ICC do not appear as three but only as two distinguishable unities. The cognitive field appears as a clearly distinguishable unity in its own right. As to the conative and affective field the outcome calls forth the question 
if the coherence that was found is, or can be made, acceptable from a psychological point of view. The two other results that stand out are the imbalance in the standard score and the low correlation between the item lists and the VAS scales that were completed. In itself these results speak a clear language. But these results also raise questions about the judgemental abilities of people and carers involved. The 'standard score' was determined by completing Part I of the ICC by one of the parents. The construct validity was determined by completing Part I of the ICC by professional coaches and by completing the VAS scales by practitioners.

\section{Limitations}

Some limitations of the research have a direct connection with the fact that the research was carried out in the practice of healthcare. For this reason the research group of children with development disorders has not been put together in a random way. Part II of the ICC, the VAS scales, could not be included in the research regarding reliability. The (average) time between the first and the second assessment regarding intra-rater reliability exceeded the two weeks that were planned and turned out to be 5.4 weeks. The average age of the control group equalled the age of the research group, but was not matched as to age structure. The COSMIN-checklist was not taken as a lead regarding determining Responsiveness.

\section{Contribution ICC}

This research contributes to the scientific foundation of the usage of constitutional typology and the ICC in healthcare. The polar formulated items and VAS scales are examples that show how a questionnaire can be drawn up that is based on a dynamic health concept.

\section{Future Perspectives}

Research is going on into the theoretical foundation of the construct of constitutional typology, which is of course necessary in the light of the results of the research. A question for further research is: How do the resulting structural validity $(<30 \%)$ and the fact that the three subscales of the ICC in the PCA only load on two factors, relate to the theory of typology?

\section{References}

American Psychiatric Association. (2013). Diagnostic and statistical manual of mental disorders (DSM-5®). American Psychiatric Pub.

American Psychiatric Association. (2015). Neurodevelopmental Disorders: DSM-5® Selections. American Psychiatric Pub.

Achenbach, T.M. (1991). Integrative guide for the 1991 CBCL/4-18, YSR, and TRF profiles. Burlington: Department of Psychiatry, University of Vermont.

Avison, W.R. (2014). Mental Illness in Childhood. The Wiley Blackwell Encyclopedia of Health, Illness, Behavior, and Society.

Baars, E. (Ed.). (2005). Goede zorg: een antroposofische benadering van ethische uitgangspunten en methodische aspecten van goede zorg. Zeist: Christofoor.

Buitelaar, J.K., \& Van Der Gaag, R.J. (1998). Diagnostic rules for children with PDD-NOS and multiple complex developmental disorder. The Journal of Child Psychology and Psychiatry and Allied Disciplines, 39(6), 911-919.

Cook, A., Spinazzola, J., Ford, J., Lanktree, C., Blaustein, M., Cloitre, M., ... Mallah, K. (2017). Complex trauma in children and adolescents. Psychiatric Annals, 35(5), 390-398.

Dai, D.Y., \& Sternberg, R.J. (Eds.). (2004). Motivation, emotion, and cognition: Integrative perspectives on intellectual functioning and development. Routledge.

Dennis, M., Simic, N., Bigler, E.D., Abildskov, T., Agostino, A., Taylor, H.G., ... Yeates, K.O. (2013). Cognitive, affective, and conative theory of mind (ToM) in children with traumatic brain injury. Developmental Cognitive Neuroscience, 5, 25-39.

Gluckman, P.D., Hanson, M.A., \& Low, F.M. (2011). The role of developmental plasticity and epigenetics in human health. Birth Defects Research Part C: Embryo Today: Reviews, 93(1), 12-18.

Goodman, A., \& Goodman, R. (2009). Strengths and difficulties questionnaire as a dimensional measure of child mental health. Journal of the American Academy of Child \& Adolescent Psychiatry, 48(4), 400-403.

Göschel, J.C. (2012) Der biografishe Mythos als pädagogisches Leitbild. Dornach: Verlag am Goetheanum.

Grimm, R., \& Kaschubowski, G. (2008). Kompendium der anthroposophischen Heilpädagogik. Basel: Reinhardt. 
Hengeveld, M.W., \& Schudel, W.J. (2004). Het psychiatrisch onderzoek (3de druk). Tijdschrift voor Psychiatrie, 46(12), 823-824.

Hilgard, E.R. (1980). The trilogy of mind: Cognition, affection, and conation. Journal of the History of the Behavioral Sciences, 16(2), 107-117.

Hochberg, Z.E., Feil, R., Constancia, M., Fraga, M., Junien, C., Carel, J.C., ... Scharfmann, R. (2010). Child health, developmental plasticity, and epigenetic programming. Endocrine Reviews, 32(2), 159-224.

Holzapfel, W. (1985). Seelenpflegebedürftige Kinder, Band I und II. Dornach: Verlag am Goetheanum.

Huber, M., Knottnerus, J.A., Green, L., Horst, H.V.D., Jadad, A.R., Kromhout, D., ... Smid, H. (2011). How should we define health? BMJ-British Medical Journal, 343(6), d4163.

Hudziak, J.J., Achenbach, T.M., Althoff, R.R., \& Pine, D.S. (2007). A dimensional approach to developmental psychopathology. International Journal of Methods in Psychiatric Research, 16(S1), S16-S23.

Kaplan, B., Crawford, S., Cantell, M., Kooistra, L., \& Dewey, D. (2006). Comorbidity, co - occurrence, continuum: what's in a name?. Child: Care, Health and Development, 32(6), 723-731.

Kraemer, H.C. (2007). DSM categories and dimensions in clinical and research contexts. International Journal of Methods in Psychiatric Research, 16(S1), S8-S15.

Luthar, S.S., Sawyer, J.A., \& Brown, P.J. (2006). Conceptual issues in studies of resilience. Annals of the New York Academy of Sciences, 1094(1), 105-115.

Mokkink, L.B., Terwee, C.B., Patrick, D. L., Alonso, J., Stratford, P. W., Knol, D. L., ... de Vet, H. C. (2012). COSMIN checklist manual. Amsterdam: University Medical Center.

Niemeijer, M.H., \& Baars, E.W. (2004) Beeldvormende diagnostiek van de kinderlijke constitutie. Driebergen: Louis Bolk Instituut.

Niemeijer, M.H., Baars, E.W., Hoekman, J., \& Ruijssenaars, A. J. J. M. (2018). An instrument for dimensional diagnosis of a child's constitution (ICC). Int J Complement Alt Med, 11(2), 68-73.

Niemeijer, M.H., \& Gastkemper, M. (2009). Ontwikkelingsstoornissen bij kinderen. Assen: Van Gorcum.

Oranje, B., Lahuis, B., van Engeland, H., van der Gaag, R.J., \& Kemner, C. (2013). Sensory and sensorimotor gating in children with multiple complex developmental disorders (MCDD) and autism. Psychiatry Research, 206(2-3), 287-292.

Pennington, B.F. (2006). From single to multiple deficit models of developmental disorders. Cognition, 101(2), $385-413$.

Pervin, L.A., \& John, O.P. (1999). Handbook of personality: Theory and research. Elsevier.

Rutter, M. (2011). Research review: child psychiatric diagnosis and classification: concepts, findings, challenges and potential. Journal of Child Psychology and Psychiatry, 52(6), 647-660.

Schafer, J. L. (1999). Multiple imputation: a primer. Statistical Methods in Medical Research, 8(1), 3-15.

Sherman, D.K., Iacono, W.G., \& McGue, M.K. (1997). Attention-deficit hyperactivity disorder dimensions: a twin study of inattention and impulsivity-hyperactivity. Journal of the American Academy of Child \& Adolescent Psychiatry, 36(6), 745-753.

Steiner, R. (1985). Heilpädagogischer Kurs. Rudolf Steiner Verlag.

Van der Kolk, B.A. (2017). Developmental Trauma Disorder: Toward a rational diagnosis for children with complex trauma histories. Psychiatric Annals, 35(5), 401-408.

\section{Copyrights}

Copyright for this article is retained by the author(s), with first publication rights granted to the journal.

This is an open-access article distributed under the terms and conditions of the Creative Commons Attribution license (http://creativecommons.org/licenses/by/4.0/). 